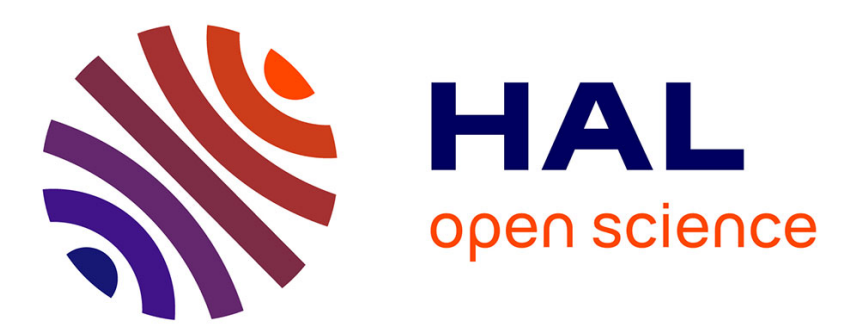

\title{
Controlling the velocities and the number of emitted particles in the tunneling to open space dynamics
}

Axel Lode, Shachar Klaiman, Ofir Alon, Alexej Streltsov, Lorenz Cederbaum

\section{To cite this version:}

Axel Lode, Shachar Klaiman, Ofir Alon, Alexej Streltsov, Lorenz Cederbaum. Controlling the velocities and the number of emitted particles in the tunneling to open space dynamics. Physical Review A, 2014, 89, pp.053620. 10.1103/PhysRevA.89.053620 . hal-02369817

\section{HAL Id: hal-02369817 https://hal.science/hal-02369817}

Submitted on 19 Nov 2019

HAL is a multi-disciplinary open access archive for the deposit and dissemination of scientific research documents, whether they are published or not. The documents may come from teaching and research institutions in France or abroad, or from public or private research centers.
L'archive ouverte pluridisciplinaire $\mathbf{H A L}$, est destinée au dépôt et à la diffusion de documents scientifiques de niveau recherche, publiés ou non, émanant des établissements d'enseignement et de recherche français ou étrangers, des laboratoires publics ou privés. 


\title{
Controlling the velocities and the number of emitted particles in the tunneling to open space dynamics
}

\author{
Axel U. J. Lode, ${ }^{1,2,{ }^{*}}$ Shachar Klaiman, ${ }^{1}$ Ofir E. Alon, ${ }^{3, \dagger}$ Alexej I. Streltsov, ${ }^{1}$ and Lorenz S. Cederbaum ${ }^{1}$ \\ ${ }^{1}$ Theoretische Chemie, Physikalisch-Chemisches Institut, Universität Heidelberg, Im Neuenheimer Feld 229, D-69120 Heidelberg, Germany \\ ${ }^{2}$ Department of Physics, University of Basel, Klingelbergstrasse 82, CH-4056 Basel, Switzerland \\ ${ }^{3}$ Department of Physics, University of Haifa at Oranim, Tivon 36006, Israel
}

(Received 16 September 2013; revised manuscript received 26 March 2014; published 19 May 2014)

\begin{abstract}
A scheme to control the many-boson tunneling process from a trap through a potential barrier to open space is devised and demonstrated. The number of ejected particles and their velocities can be controlled by two parameters, the threshold of the potential and the interparticle interaction. Employing a recently developed efficient many-body method, results for two, three, and one hundred interacting bosons in one spatial dimension can be computed numerically exactly, solving thereby an intricate problem of general interest. It is shown that the control scheme devised for the many-boson tunneling process performs very well for the dynamics of the momentum density, the correlations, and the coherence, as well as for the number of particles remaining in the trap. To interpret the many-body tunneling process, a transparent model assembling the many-body process from single-particle emission processes is derived. Analysis of the energetics of available decay channels together with the numerical results rules out the situation of two (or more) bosons tunneling together to open space. The present investigation generalizes the findings reported by us [Proc. Natl. Acad. Sci. USA 109, 13521 (2012)] for many-boson tunneling to open space in the absence of a threshold.
\end{abstract}

DOI: 10.1103/PhysRevA.89.053620

PACS number(s): 03.75.Kk, 03.65.-w, 05.30.Jp, 03.75.Lm

\section{INTRODUCTION}

The understanding of quantum many-body dynamics has been pushed forward in recent years by the realization of unique possibilities for controlling Bose-Einstein condensates (BECs) in the laboratory [1-5]. The confinement of BECs [6], their interparticle interactions [7], and their dimensionality [8-10] can be manipulated in experiments almost at will. Using these extensive mechanisms of control, BECs are used as so-called quantum simulators to study a variety of physical systems: Solid-state systems are studied with optical lattices [1,11-13] and even problems in astrophysics are tackled [14-16].

One of the fundamental phenomena of quantum mechanics is the tunneling process. The process of tunneling to open space in many-particle systems appears in nature in a variety of systems, like $\alpha$ decay, fusion, and fission in nuclear physics as well as photoassociation and photodissociation in biology and chemistry [17-21]. Despite lacking the energy to overcome a potential barrier, quantum particles are able to escape by tunneling through the barrier. This is due to the probabilistic nature of quantum mechanics: The particles have a nonzero probability to be found on the other side of the barrier. The physics of tunneling for a single particle is well understood [22] and was described already in the 1920s (see Refs. [23-25]). The corresponding many-body process with interacting particles is naturally more involved and has also been studied (see, e.g., Refs. [26-35]). In the case of many-body tunneling, the mechanism of the dynamics has only recently been revealed [35]. Specifically, it has been shown in Ref. [35] how the emitted particles dissociate or fragment from a trapped and coherent source of bosons. The overall

\footnotetext{
*axel.lode@unibas.ch

${ }_{\dagger}^{\dagger}$ ofir@ research.haifa.ac.il
}

many-boson decay dynamics is built up as an interference of many simultaneous single-particle emission processes. The velocities of the emitted bosons are dictated to a large accuracy by the chemical potentials of trapped interacting systems of different particle numbers.

A central theme of the tunneling of a BEC to open space is that the dynamics is a many-body process [35]. Explicitly, the system gradually loses its initial coherence and becomes a fragmented BEC. A many-boson system is said to be fragmented [36-42] if it occupies more than one single-particle state. Therefore, such a fragmented system is also not a coherent one, in the sense that its correlation functions [43-46] are not flat. In the tunneling process, the emitted particles lose the coherence with the trapped source and among each other. We stress that the above many-body features are caused by the interactions of the particles. For a noninteracting system the coherence of the bosons is fully maintained throughout space and the tunneling system does not fragment.

In the present work it is shown that the many-boson tunneling to open space dynamics can be controlled extensively by introducing a potential threshold. To investigate the many-body physics of a BEC tunneling to open space from a trap with a potential threshold we employ, as in Ref. [35], the multiconfigurational time-dependent Hartree for bosons (MCTDHB) method [47-49]. The MCTDHB method provides the means to solve the time-dependent many-boson Schrödinger equation numerically accurately for a wide range of problems (see, e.g., Refs. [35,50-55]) and is well documented in the literature [56,57].

The structure of the paper is as follows. In Sec. II we present the setup of the tunneling process including the threshold potential, and in Sec. III we discuss the analysis tools including a simplified model employed in this work. In Sec. IV we investigate the control of the tunneling process by varying the threshold, and in Sec. V its control by varying the interparticle interaction. An application to the decay by tunneling of a 
101-boson system is presented in Sec. VI. We summarize in Sec. VII. Technical details of the setup and computation are deferred to the Appendixes.

\section{HAMILTONIAN AND THE SETUP}

The tunneling process of ultracold many-body systems to open space is described by the time-dependent Schrödinger equation,

$$
i \partial_{t}|\Psi\rangle=\hat{H}|\Psi\rangle .
$$

Here, $|\Psi\rangle$ is the wave function which depends on the spatial coordinates of all particles and $\hat{H}$ the many-body Hamiltonian,

$$
\hat{H}=\sum_{i=1}^{N} \hat{h}_{i}+\sum_{i<j=1}^{N} \hat{W}_{i j} .
$$

For ultracold atomic bosons $\hat{H}$ contains one-body operators $\hat{h}_{i}$ for each boson and two-body operators $\hat{W}_{i j}$ for every pair of particles. The one-body Hamiltonian $\hat{h}_{i}$,

$$
\hat{h}_{i}=-\frac{1}{2} \partial_{x_{i}}^{2}+V\left(x_{i}\right),
$$

contains the kinetic energy $-\frac{1}{2} \partial_{x_{i}}^{2}$ and the one-body threshold potential $V\left(x_{i}\right)$. Figure 1 depicts schematically the setup of the system to be studied in the present work. The technical design and properties of the potential $V(x)$ are discussed in Appendix A.

Throughout this work we employ dimensionless units. This means that $\hat{H}$ has been divided by $\frac{\hbar^{2}}{L^{2} m}$, where $\hbar$ is Planck's constant, $m$ is the mass of a boson, and $L$ is a length scale that one introduces. As the two-body interaction we use the standard contact interaction potential $\hat{W}_{i j}=\lambda_{0} \delta\left(x_{i}-x_{j}\right)$. The parameter $\lambda_{0}=\frac{2 m \omega_{\perp} L}{\hbar} a_{s}$ is related to the $s$-wave scattering

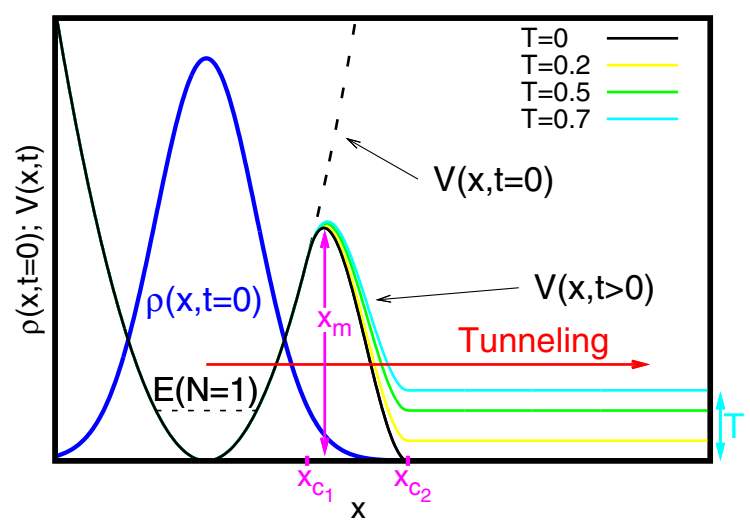

FIG. 1. (Color online) Protocol for tunneling dynamics with nonzero potential threshold. The initial density [blue (gray) solid line above $\rho(x, t=0)$ ] is prepared as the ground state of the parabolic trap [black dashed $V(x, t=0)$ ]. Subsequently, the potential is transformed to its open form with a threshold [various solid colored (gray) lines $V(x, t>0)]$. Following this transformation the particles can tunnel to open space. The tunneling process can be controlled by the threshold $T$. The energy of a single, parabolically trapped particle, $E(N=1)$, is indicated by the horizontal black dashed line to guide the eye. See the text and Appendix A for more details. All quantities shown are dimensionless. length $a_{s}$ in the ultracold atomic sample. It can be tuned with the aid of Feshbach resonances [7] or the trap geometry, i.e., the transverse frequency $\omega_{\perp}$ of the confinement.

To solve the many-boson Schrödinger equation (1) we employ the MCTDHB method [47-49]. The key idea in its efficiency and capability to solve Eq. (1) numerically exactly [54] lies in the usage of a time-dependent, variationally optimized many-body basis set. Since there is already extensive literature on the MCTDHB method (see, e.g., Refs. [56,57]), we have deferred to Appendix B the expository and technical details relevant to the present investigations.

\section{TOOLS OF ANALYSIS}

\section{A. Fragmentation, probability, and coherence}

The full wave function which is available in the MCTDHB computations at any given point in time is a complicated and high-dimensional quantity. To investigate the many-body state and its dynamics it is thus desirable to have a set of appropriate quantities for the analysis. It is a standard practice to employ the reduced one-body density matrix for the purposes of both analysis and visualization [45,46,58]. The reduced one-body density matrix is defined as

$$
\begin{aligned}
\rho^{(1)}\left(x_{1} \mid x_{1}^{\prime} ; t\right)= & N \int \Psi\left(x_{1}, \ldots, x_{N} ; t\right) \Psi^{*}\left(x_{1}^{\prime}, x_{2}, \ldots, X_{N} ; t\right) \\
& \times d x_{2} \cdots d x_{N} .
\end{aligned}
$$

When one expands it in its eigenfunctions, the so-called natural orbitals $\left\{\phi_{i}(x, t) ; i=1, \ldots, M\right\}$, it takes on the form

$$
\rho^{(1)}\left(x_{1} \mid x_{1}^{\prime} ; t\right)=\sum_{i} \rho_{i}^{(N O)}(t) \phi_{i}\left(x_{1}, t\right) \phi_{i}^{*}\left(x_{1}^{\prime}, t\right) .
$$

The natural occupations $\rho_{i}^{(N O)}(t)$ and the natural orbitals, as well as the density $\rho(x, t) \equiv \rho^{(1)}\left(x_{1}=x \mid x_{1}^{\prime}=x ; t\right)$ are very useful quantities for assessing quantum many-boson dynamics. From the natural occupations one can infer if a system is condensed or fragmented. If only a single macroscopic eigenvalue $\rho_{1}^{(N O)}$ is present then the system is referred to as condensed [59]. If multiple eigenvalues $\left\{\rho_{i}^{(N O)} ; i=1, \ldots, M\right\}$ are macroscopic, then the system is referred to as fragmented [36-42].

The density $\rho(x, t)$ describes the probability of finding a single particle in the many-body system at a certain position $x$ at a given time $t$. As a measure of the number of particles remaining inside the parabolic part of the potential (see Fig. 1), it is instructive to define the nonescape probability

$$
P_{\text {not }}^{x}(t, T)=\int_{-\infty}^{x_{m}} \rho(x, t) d x
$$

$\left[x_{m}\right.$ is where the potential barrier gets its maximum; see Fig. 1 and Eq. (A2)].

The coherence of the quantum many-body state can be analyzed with the aid of Glauber's first-order normalized correlation function [43-46],

$$
g^{(1)}\left(x_{1}^{\prime}, x_{1} ; t\right)=\frac{\rho^{(1)}\left(x_{1} \mid x_{1}^{\prime} ; t\right)}{\sqrt{\rho\left(x_{1}, t\right) \rho\left(x_{1}^{\prime}, t\right)}} .
$$

If $\left|g^{(1)}\right|^{2}=1$ holds, the system is coherent. This is true only for the case when $\rho^{(1)}$ is built up as a product of a single 
complex-valued function (see, e.g., Ref. [44]). The latter implies that $\rho^{(1)}$ has only a single eigenvalue $\rho_{1}^{(N O)}$ and hence $\left|g^{(1)}\right|^{2}=1$ also means that the system is fully condensed. On the other hand, $\left|g^{(1)}\right|^{2}<1$ implies that $\rho^{(1)}$ is built up from several complex-valued functions and has several contributing eigenvalues $\left\{\rho_{i}^{N O}(t) ; i=1, \ldots, M\right\}$. Hence, $\left|g^{(1)}\right|^{2}<1$ also implies a departure from coherence and the occurrence of depletion and, eventually, of fragmentation.

All the above quantities can also be transformed to momentum space (see, e.g., Ref. [46]). The momentum-space representation is particularly versatile for assessing quantum tunneling dynamics [35], and will frequently be employed throughout the present study.

\section{B. A simple model as interpretational tool}

Consider the system as split into an "IN" part, to the left of the maximum of the barrier, and an "OUT" part to the right of it (see Fig. 2). The IN part is the part of the potential that is classically allowed, i.e., classical particles would be indefinitely confined in the IN region.

Suppose a single boson has escaped from the IN to the OUT region. Following the analysis in Ref. [35], the available energy of this boson must come from the energy difference of the trapped systems with $N$ and with $N-1$ particles, $E^{N}-E^{N-1}=\mu_{1}$ - the chemical potential of the $N$-particle system. With this in principle available energy, the ejected boson has to overcome the threshold $T$. Hence, it remains with an energy $\left(\mu_{1}-T\right)$ in the OUT part of the potential. As the potential in the OUT part is flat and the density can be assumed to be small, the ejected boson will convert its available energy to kinetic energy. Analogously, the other particles which are ejected have their available energies from the chemical potentials $\mu_{i}$ of $(N-i)$ particles left in the trap. One can hence derive momenta $k_{i}$ from the related kinetic

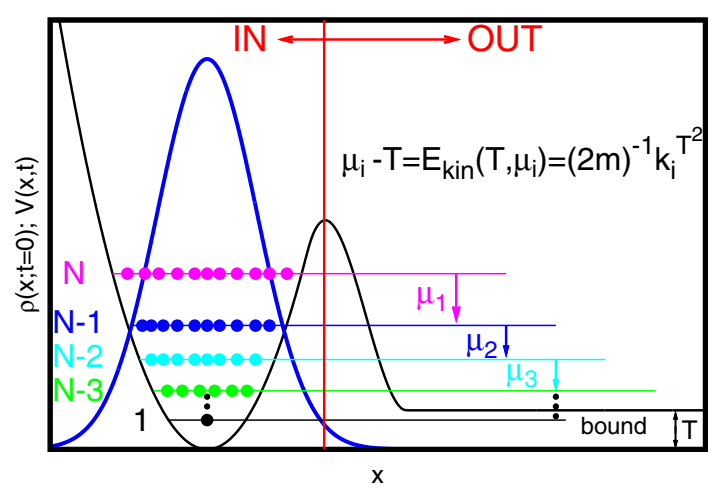

FIG. 2. (Color online) Static mean-field scheme to model the tunneling processes with a threshold $T$. The bosons are tunneling from the interior IN to the exterior OUT region of space (separated by the vertical red line). If the threshold $T$ is large enough, some of the states can become bound (see, e.g., the $N=1$ state indicated by the lowest black line). If the state is not bound, the chemical potential $\mu_{i}$ is used to overcome the threshold $T$ and the remainder is transformed to kinetic energy $E_{\text {kin }}\left(T, \mu_{i}\right)$. The momenta $k_{i}=$ $\sqrt{2 m\left[E_{\mathrm{kin}}\left(T, \mu_{i}\right)\right]}=\sqrt{2 m\left(\mu_{i}-T\right)} ; i=N, N-1, \ldots, 1$ appear in the momentum distribution; see the arrows in Fig. 4 and dashed lines in Fig. 5 below. All quantities shown are dimensionless. energies:

$$
\begin{aligned}
E_{\mathrm{kin}}\left(T, \mu_{i}\right) & =\mu_{i}-T=\frac{\left(k_{i}^{T}\right)^{2}}{2 m} \Rightarrow \\
k_{i}^{T} & =\sqrt{2 m\left[E_{\mathrm{kin}}\left(T, \mu_{i}\right)\right]}=\sqrt{2 m\left(\mu_{i}-T\right)} .
\end{aligned}
$$

The model Eq. (8) assumes that there is no interaction between the particles in the OUT region. In the absence of any interaction, all chemical potentials are equal, i.e., $\mu_{1}=\mu_{2}=\cdots=\mu_{N}$. The predictions of the simple model Eq. (8) will be tested against the numerically exact many-body treatment in Sec. IV. As a result, the model will be validated and shown to define the modalities of the tunneling process; see the momentum distributions shown in Fig. 4 below.

An important feature of the class of potentials with a nonzero asymptotic value is that they can have bound states. If one raises the threshold $T$ beyond the chemical potential $\mu_{i}$ of a certain trapped bosonic system, then the bosons in systems with $\mu_{i}<T$ do not have enough energy to overcome $T$ and therefore stay trapped. Hence, the IN system is in a bound state [see Fig. 2 and Eq. (8)]. This property of the trap suggests that one can control the number of bound particles with both the interaction $\lambda_{0}$ and the threshold $T$ itself. By manipulating the interaction $\lambda_{0}$ the energies and especially the chemical potentials can be controlled, and by adjusting the threshold $T$ the number of bound particles can be adjusted. In the case of vanishing interaction, the threshold $T$ controls whether the whole system is bound or not.

In what follows we introduce the notation $\left|N_{\mathrm{IN}}, N_{\mathrm{OUT}}\right\rangle$, where $N_{\text {IN }}$ counts the number of particles in the IN region and $N_{\text {OUT }}$ in the OUT region, according to the partition in Fig. 2. Henceforth, the introduced notation will be referred to as the counting statistics of a given state (and should not be confused with a Fock state). Among others, we will use the $\left|N_{\text {IN }}, N_{\text {OUT }}\right\rangle$ quantity to analyze numerically computed nonescape probabilities [see Eq. (6)].

What is the minimal energy for sustaining a state with counting statistics $\left|N_{\text {IN }}, N_{\text {OUT }}\right\rangle$ ? The energy for the IN particles is essentially given by the energy of $N_{\mathrm{IN}}$ interacting bosons in a parabolic potential. We denote this energy by $E_{\mathrm{HO}}\left(N_{\mathrm{IN}}, \lambda_{0}\right)$, indicating that it explicitly depends on the number of particles $N_{\text {IN }}$ and the interaction between them $\lambda_{0}$. The minimal energy for the OUT particles is essentially given by $N_{\text {OUT }}$ bosons at rest at the threshold potential energy $T$, namely, by $N_{\text {OUT }} T$. It follows that the minimal energy obeys the relation

$$
E_{\mathrm{TOT}}\left(N_{\mathrm{IN}}, N_{\mathrm{OUT}}, T, \lambda_{0}\right)=E_{\mathrm{HO}}\left(N_{\mathrm{IN}}, \lambda_{0}\right)+N_{\mathrm{OUT}} T .
$$

To summarize, one can adjust the energies of the bosons inside the trap by tuning the interaction $\lambda_{0}$ and the energies of the bosons outside the trap by tuning the threshold $T$. These two possibilities are explored and demonstrated in the following two sections.

\section{CONTROLLING THE DYNAMICS BY VARYING THE THRESHOLD}

\section{A. Dynamics of the tunneling process}

For transparency we study the impact of changing the threshold on the simplest possible many-body system, i.e., $N=2$ interacting bosons. As we shall see, their dynamics 

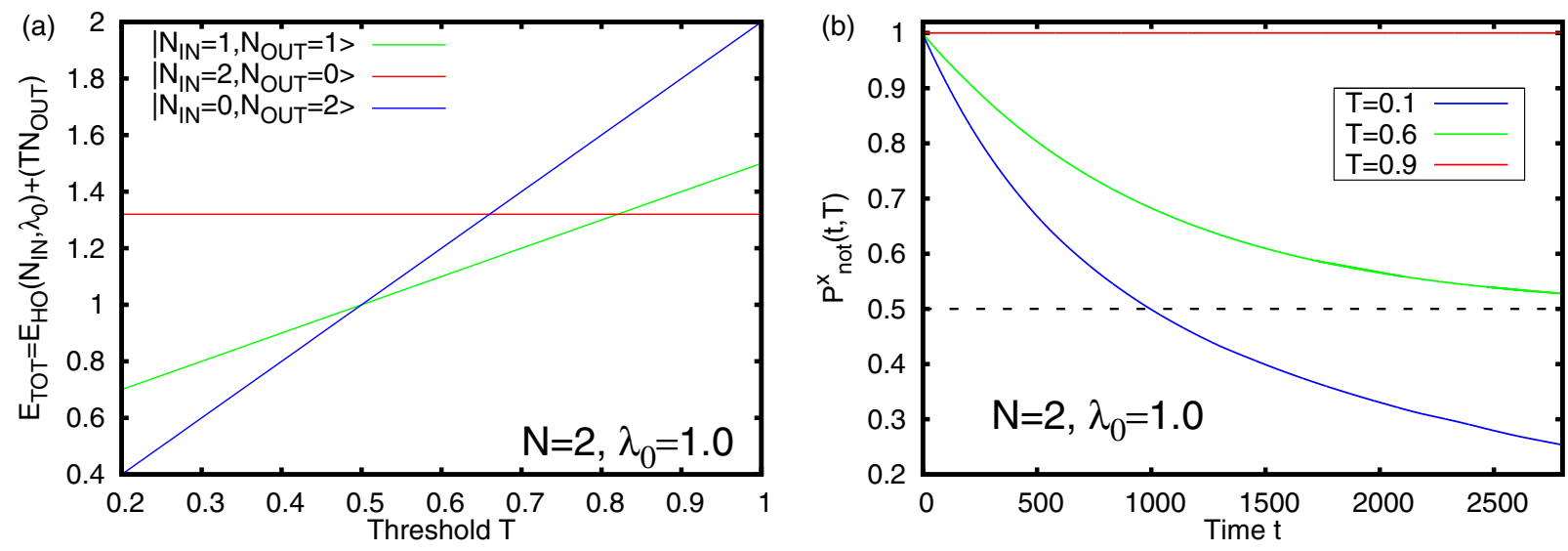

FIG. 3. (Color online) Controlling the two-boson tunneling dynamics by varying the threshold. (a) The energies $E_{\mathrm{TOT}}$ of the possible final states $\left|N_{\text {IN }}, N_{\text {OUT }}\right\rangle=|2,0\rangle,|1,1\rangle,|0,2\rangle$ for $\lambda_{0}=1.0$ as functions of $T$. At $T=0.5$, a one-particle bound state emerges in the trap and at $T \approx 0.8$ the two-boson system becomes bound. The crossing points of $E_{\mathrm{TOT}}$ define critical thresholds where the energetically favorable final state of the dynamics is changing. (b) The nonescape probabilities $P_{\text {not }}^{x}(t, T)$ for different thresholds $T=0.1,0.6,0.9$ plotted from bottom to top as the blue, green, and red solid lines, respectively. For $T=0.1$ the final state $|0,2\rangle$ is favorable whereas for $T=0.6$ it is $|1,1\rangle$. The horizontal dashed line marks $P_{\text {not }}^{x}=0.5$, the nonescape probability of the final state $|1,1\rangle$. For $T \gtrsim 0.8$, the two-boson system is bound. See the text for further discussion. All quantities shown are dimensionless.

is extremely rich. We begin with an energy analysis of the possible states in the system. For this, it is instructive to fix the interaction at a certain value and vary only the threshold of the potential. Figure 3(a) shows the energies Eq. (9) of the possible final states for $\lambda_{0}=1.0$ as a function of $T$.

The respective lowest line segment in Fig. 3(a) indicates the energetically favorable final state for the dynamics. Hence, the crossing points of the lines define critical thresholds: At these values of $T$ the energetically favorable final state of the dynamics is changing. One would thus expect that for $T \leqslant 0.5$ both particles decay whereas for $0.5<T \lesssim 0.8$ one particle decays and one stays bound. For $T \gtrsim 0.8$ the whole system is bound and no particle decays. Since the nonescape probability $P_{\text {not }}^{x}(t, T)$ counts $\frac{N_{\mathrm{IN}}}{N}$ particles inside the trap, it should tend to 0 for the $|0,2\rangle$ final state, to $\frac{1}{2}$ for the $|1,1\rangle$ final state, and stay at 1 for the bound $|2,0\rangle$ final state. To verify this behavior, Fig. 3(b) shows a plot of the nonescape probabilities for the thresholds $T=0.1,0.6$, and 0.9 . The results demonstrate how the threshold can be used to control the final state of the system by modifying the energy of the OUT particles, $N_{\text {OUT }} T$. Explicitly, by tuning $N_{\text {OUT }} T$ beyond the largest chemical potential of an $N_{\mathrm{IN}}$-body system, one creates an $N_{\text {IN }}$-body bound state and $N_{\text {OUT }}$ particles which have tunneled out. This scheme allows for a flexible control of the counting statistics in the IN and OUT parts of space.

We have so far examined the control over the two-boson tunneling process in coordinate space. It is instructive to investigate the complementary picture in momentum space. Figure 4 plots the momentum distribution $\rho(k, t, T)$ at $t=600$ for several values of the threshold $T=0.0,0.1, \ldots, 0.6$. It is clearly seen that the emitted particles form a peak structure in the momentum distributions.

We recall that in the case of zero threshold, $T=0$, each emitted particle shows up as a distinct peak in the momentum density [35]. Furthermore, the momenta of the emitted particles, $k_{1}, k_{2}, \ldots$ are essentially time independent and determined by the chemical potentials $\mu_{1}, \mu_{2}, \ldots$ of systems with decreasing particle numbers $N, N-1, \ldots$ What are the changes when a threshold is introduced? First, with an increase of $T$ each peak is shifted towards $k=0$, as the escaping bosons have to invest a larger part of their available energy to overcome the higher threshold [see Eq. (8)]. Second, the bigger the threshold, the smaller is the intensity of the $k_{1}^{T}$ peak, i.e., the peak in the momentum distribution with the largest $k$ value. This means that the increase of the threshold decreases the pace with which the first boson is escaping. A similar reasoning can be applied to the second

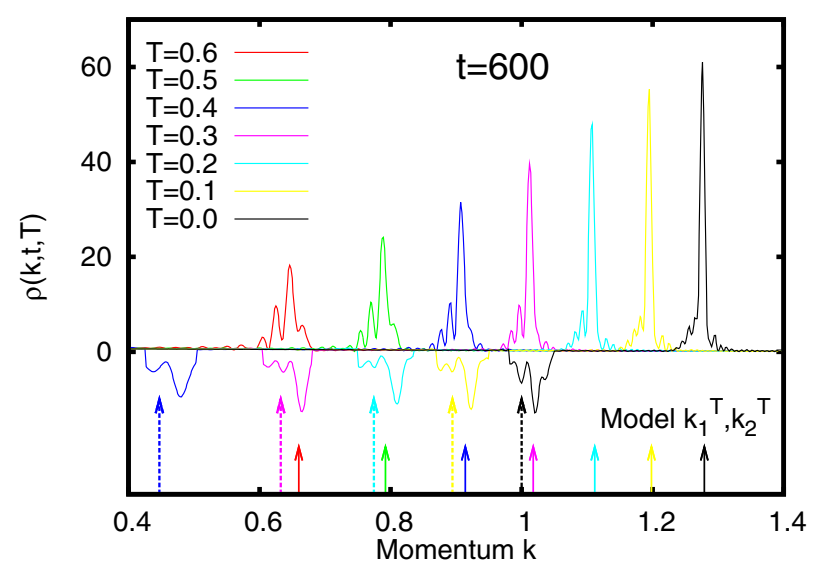

FIG. 4. (Color online) Effect of the threshold $T$ on the momentum distribution of $N=2$ interacting bosons tunneling to open space. Shown is $\rho(k, t, T)$ at $t=600$ for $T=0.0,0.1, \ldots, 0.6$ (peaks from right to left, respectively). The solid (dashed) arrows at the bottom indicate the first (second) momenta $k_{1}^{T}\left(k_{2}^{T}\right)$ obtained from the model Eq. (8). With increasing $T$ the momentum peaks are shifted towards 0 and their intensity decreases. To visualize the results, the second, less intense $k_{2}^{T}$ peaks are shown upside down and scaled by a factor of 4 for better visibility. See the text for further discussion and a complementary video in the Supplemental Material [60]. All quantities shown are dimensionless. 


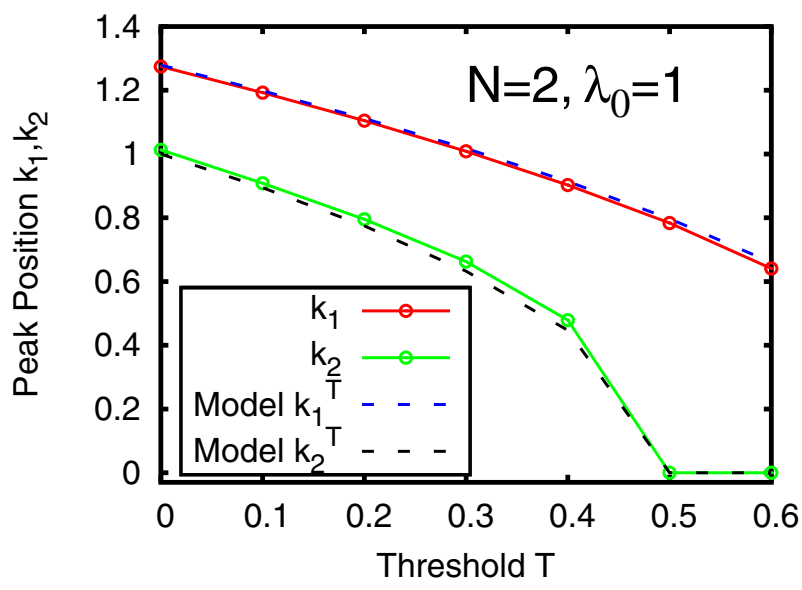

FIG. 5. (Color online) Comparison of the $N=2$ momentumpeak positions. The solid upper red and solid lower green lines with points show the positions of the peaks $k_{1}$ and $k_{2}$ obtained from the numerically exact momentum distributions, whereas the upper blue and lower black dashed lines show the predictions of the model Eq. (8) for $k_{1}^{T}$ and $k_{2}^{T}$. For the exact solutions circles represent actual data; the lines are drawn to guide the eye. See the text for further discussion. All quantities shown are dimensionless.

$k_{2}^{T}$ peak, although its intensity decreases more moderately in comparison with the $k_{1}^{T}$ peak (see Fig. 4).

The agreement of the positions of the peaks in $k$ space with the predictions of the model Eq. (8) is very good (see the arrows in Fig. 4). To further demonstrate the validity of Eq. (8) also for the second peak at $k_{2}^{T}$, we compare the numerically exact results and model's predictions as a function of the threshold in Fig. 5. The agreement is clearly seen. Consequently, one can interpret the tunneling process of the two-boson system as an interference of two single-boson tunneling processes. In the $\left|N_{\text {IN }}, N_{\text {OUT }}\right\rangle$ nomenclature they are represented as follows: $|2,0\rangle \rightarrow|1,1\rangle$ and $|1,0\rangle \rightarrow|0,1\rangle$. These single-boson processes are happening simultaneously, in analogy to the situation in the absence of a threshold [35].

In summary, the two-body tunneling dynamics to open space can be controlled by modifying the threshold in the following ways: First, the counting statistics can be controlled via the threshold by creating bound states. Second, the emergence of a bound state can be used as a control on the momentum spectra of the emitted bosons. Peaks can be shifted or even switched off (on) by making the corresponding single-boson process energetically inaccessible (accessible).

\section{B. Fragmentation, correlations, and coherence dynamics}

We have described and investigated in the previous section the impact of the threshold on tunneling to open space by examining two natural quantities: the coordinate- and momentum-space densities. We would now like to get a deeper look into the effects of the threshold on the tunneling process by recruiting other many-body analysis tools.

We begin with fragmentation. The time evolution of the occupation numbers is plotted in Fig. 6 for $N=2$ bosons with $\lambda_{0}=1.0$ for thresholds $T=0.0,0.1,0.2, \ldots, 0.6$. We find that

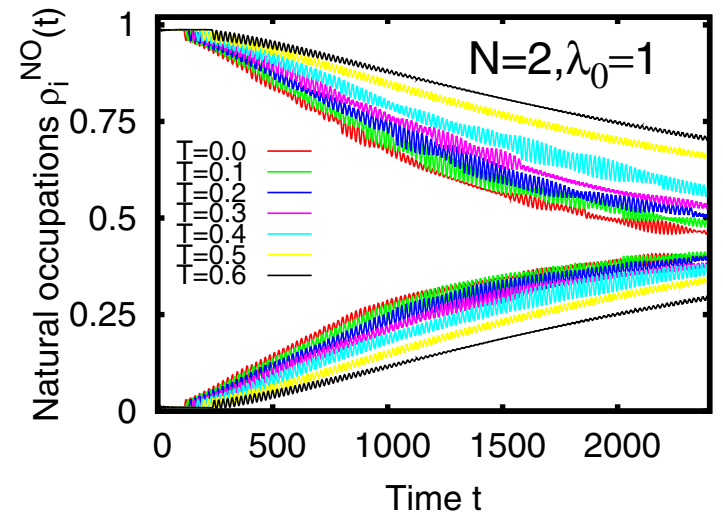

FIG. 6. (Color online) Fragmentation is delayed by increasing the threshold. Shown are the time evolutions of the first two occupation numbers $\rho_{1}^{(N O)}(t)$ and $\rho_{2}^{(N O)}(t)$ for $N=2$ interacting bosons with $\lambda_{0}=1.0$ for different thresholds $T$ [the threshold $T$ decreases (increases) from the topmost (bottommost) black curve]. The buildup of initial depletion and eventual fragmentation are delayed for larger $T$. See the text for further discussion. All quantities shown are dimensionless.

the occupation numbers depend on $T$ as follows: As the pace of the tunneling process is slowed down by the onset of the threshold, the occurrence of fragmentation is also delayed. Furthermore, the initial depletion of the system is delayed, i.e., $\rho_{1}^{(N O)} \approx 1$ holds for a longer initial time when $T$ is larger (see Fig. 6). It is interesting to note that the necessity for a multiconfigurational description persists also in the cases of $T>0.5$, i.e., when a one-boson bound state emerges and the counting statistics of the final state changes from $|0,2\rangle$ to $|1,1\rangle$. This can be inferred from the gradual (continuous) buildup of fragmentation also for this value of the threshold (see Fig. 6).

To explore the effect of the threshold on the coherence during the tunneling to open space process, one has to inspect the normalized single-particle correlation function $g^{(1)}$. In Fig. 7 a plot of $g^{(1)}$ in momentum space is given for $T=0.0,0.4,0.6$. It is seen that the correlation functions

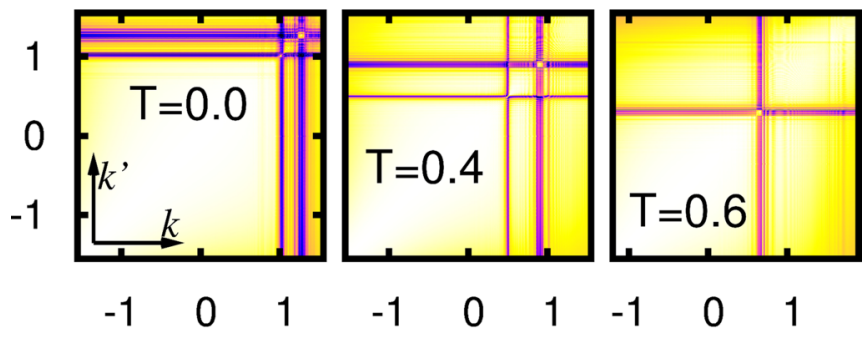

FIG. 7. (Color online) First-order coherence in the tunneling to open space of $N=2$ interacting bosons with a threshold. Shown is the single-particle momentum correlation function $\left|g^{(1)}\left(k^{\prime}, k ; t\right)\right|^{2}$ at time $t=600$ and interaction $\lambda_{0}=1.0$ for $T=0.0,0.4,0.6$. White corresponds to full first-order coherence, i.e., $\left|g^{(1)}\right|^{2}=1$, and black to full first-order incoherence, i.e., $\left|g^{(1)}\right|^{2}=0$. The ejected particles lose their coherence with the source and with each other. The change of the final state is manifest in the absence of a second line where coherence is lost $(T=0.6)$. See the text for further discussion. All quantities shown are dimensionless. 
exhibit lines of incoherence (the darker lines atop of the white and light yellow background). The positions of these lines coincide with the momenta $k_{1}$ and $k_{2}$ discussed above, thus indicating the single-particle processes assembling the manyboson tunneling. During the time evolution the positions of the lines and hence the overall structure of $g^{(1)}$ do not change, and it is therefore sufficient to depict $g^{(1)}$ at a single point in time.

The system's counting statistics changes from $|0,2\rangle$ to $|1,1\rangle$ when the threshold is increased past $T=0.5$, i.e., only one of the two particles is decaying to open space. This change manifests itself in $g^{(1)}$ by the disappearance of the line at $k_{2}$ corresponding to the now energetically forbidden process (see Fig. 7). Furthermore, with increasing $T$ the loss of coherence around the slower momentum $k_{2}$ is gradually diminished and, eventually, it becomes fully coherent (see the $T=0.6$ panel in Fig. 7). It is interesting that the coherence of the system is lost also in the cases where only a single particle is ejected. In this manner, peak after peak, corresponding to the singleparticle processes depicted in Fig. 2, the system returns to full coherence, as soon as the corresponding tunneling channel becomes energetically unfavorable.

To summarize, the dynamics of the tunneling process to open space can be managed via the threshold $T$. The occurrence of bound states manifests itself in the closing of decay channels and corresponding changes of the counting statistics in the dynamics. The momenta in the decay process are obtained from the respective chemical potentials of systems with smaller particle numbers and the threshold. The ejected particles lose coherence between each other and with the source, similarly to and extending thereby the results found in the absence of a threshold (see Ref. [35]).

\section{CONTROLLING THE DYNAMICS BY VARYING THE INTERACTION}

\section{A. Energetics and counting statistics}

Another possibility for controlling the tunneling dynamics of interacting bosons is to fix the threshold $T$ of the potential and change the strength of the interaction $\lambda_{0}$. We will explore and discuss the underlying physics for $N=3$ bosons. The system is still small enough to assure transparency, but allows one to have also a situation where after tunneling interacting particles remain in the trap. Importantly, the availability of several decay channels will allow us to cast more light on the mechanism of the tunneling process.

For $N=3$ particles, there are four possibilities for the counting statistics of the final state: $\left|N_{\text {IN }}, N_{\text {OUT }}\right\rangle=$ $|3,0\rangle,|2,1\rangle,|1,2\rangle$, and $|0,3\rangle$. We start with a discussion of the energies of these states. Figure 8(a) depicts the energies Eq. (9) of the four $\left|N_{\text {IN }}, N_{\text {OUT }}\right\rangle$ states for $T=0.7$ as functions of $\lambda_{0}$. The energies of $|3,0\rangle$ and $|2,1\rangle$ depend on $\lambda_{0}$. Obviously, the larger the number of bosons inside the trap $\left(N_{\mathrm{IN}}\right)$, the steeper is the slope of the respective energy [see Fig. 8(a)]. The energies of both $|1,2\rangle$ and $|0,3\rangle$, given by Eq. (9) as 1.9 and 2.1 , respectively, do not depend on $\lambda_{0}$. The reason is that the interaction of the emitted bosons in the OUT region is neglected in the model. These are the minimal energies the system needs in order to eject two and three particles, respectively.
Since the energies of the four possible final states $\left|N_{\text {IN }}, N_{\text {OUT }}\right\rangle$ depend differently on $\lambda_{0}$, they can be expected to cross each other. We find five such intersections, marking critical interaction strengths where the counting statistics changes, and label them by $\lambda_{c_{i}}, i=1, \ldots, 5$, in Fig. 8(a). Obviously, if one chooses an interaction $\lambda_{0}$ smaller than the first critical interaction $\lambda_{c_{1}}$, then the initially prepared three-boson system remains bound. There is simply not enough (interaction) energy to overcome the threshold $[T=0.7$ in Fig. 8(a)]. If one chooses $\lambda_{c_{1}}<\lambda_{0}<\lambda_{c_{2}}$, then the energy of the state $|2,1\rangle$ is lower than that of $|3,0\rangle$. We may thus anticipate that the initially prepared three-boson system decays in time to $|2,1\rangle$. Next, if one chooses $\lambda_{c_{2}}<\lambda_{0}<\lambda_{c_{3}}$ then, according to Fig. 8(a), there are two states- $|2,1\rangle$ and $|1,2\rangle$-with energies lower than that of $|3,0\rangle$. Because the energy of the final state $|2,1\rangle$ is lower than that of $|1,2\rangle$, we incline to expect that the initially prepared three-boson system will decay to the former state. We will put this conjecture to the test in the following section, when the dynamics is performed and investigated. One can apply a similar reasoning to the other critical interactions $\lambda_{c_{4}}$ and $\lambda_{c_{5}}$. It is now time to investigate the counting statistics in the actual many-body tunneling dynamics process.

\section{B. Dynamics and properties of the tunneling process}

Motivated by the energetics analysis of the previous section, we proceed to investigate the dynamics. Figure 8(b) presents the nonescape probabilities $P_{\text {not }}^{x}(t, T)$ of $N=3$ bosons in a potential with a fixed threshold $T=0.7$ for three interactions $\lambda_{0}$.

We begin with $\lambda_{0}=0.5$. Inspection of Fig. 8(b) reveals unequivocally that the nonescape probability $P_{\text {not }}^{x}(t, T)$ converges to $\frac{2}{3}$. This means that the counting statistics of the tunneling process amounts to the state $|2,1\rangle$. We recall from the above discussion and energetics analysis [see Fig. 8(a)], that there are in principle two states- $|2,1\rangle$ and $|1,2\rangle$-available for the tunneling dynamics for $\lambda_{0}=0.5$. Why does the counting statistics converge to the lowest-in-energy state $|2,1\rangle$ and not to the available, higher-energy state $|1,2\rangle$ ? What can we learn from the results for the dynamics of the nonescape probability? First and importantly, that the tunneling process of the bosons to open space does not occur in pairs. For if two bosons were to tunnel together, they would have stayed outside, and the counting statistics becomes the state $|1,2\rangle$. Furthermore, once the first boson starts to tunnel, the second one does not have enough energy to follow it. In other words, we can infer from Fig. 8(a) that, for the system of two bosons remaining after the first boson has tunneled out, the two-boson state $|2,0\rangle$ is lower in energy than the state $|1,1\rangle$. Consequently, no second boson can tunnel out for $\lambda_{0}=0.5$. This will also be evident in the momentum correlations discussed below. In conclusion, the interpretational picture of the tunneling process of interacting bosons as an interference of one-particle tunneling processes (see Fig. 2) gets further support from these findings.

For $\lambda_{0}=1.0$ the nonescape probability $P_{\text {not }}^{x}(t, T)$ converges to $\frac{1}{3}$ [see Fig. 8(b)]. Again, out of the three energetically available states- $|0,3\rangle,|2,1\rangle$, and $|1,2\rangle$ (in descending order of energy) - the system converges to the lowest-in-energy state $|1,2\rangle$. We can thus infer the following mechanism: The initial three-boson system ejects the first boson; this already 

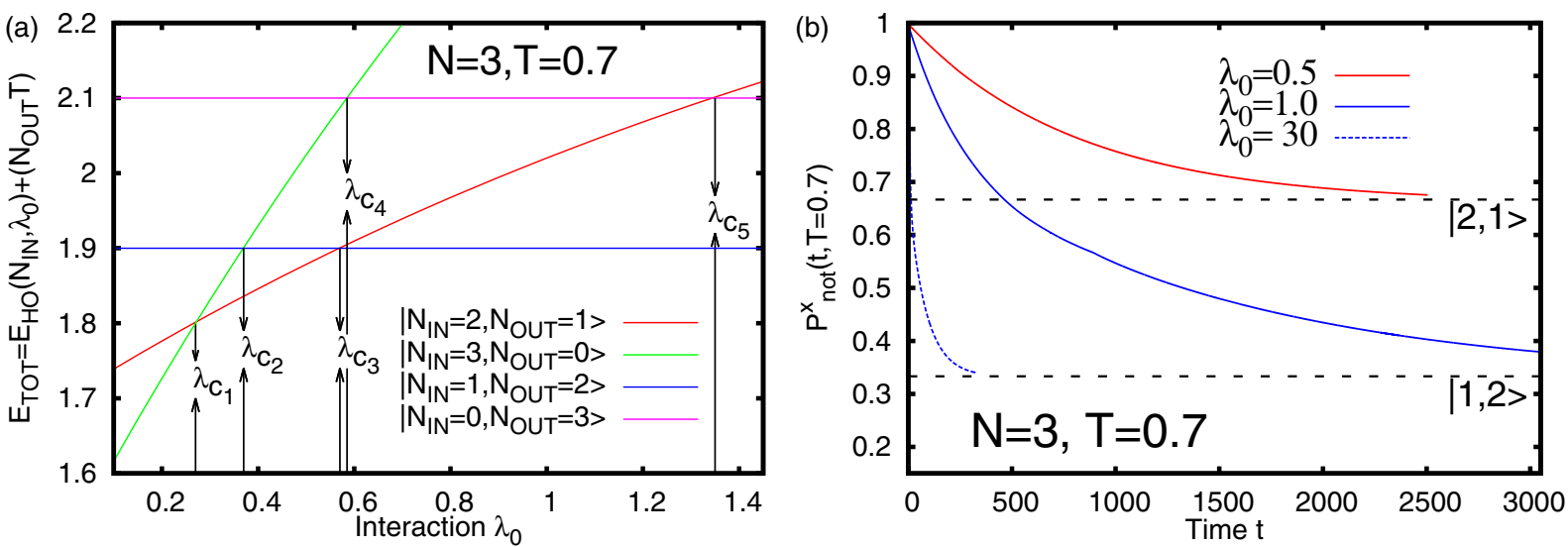

FIG. 8. (Color online) Controlling the tunneling dynamics of $N=3$ bosons by varying the interaction. (a) The energies of the possible final states $\left|N_{\text {IN }}, N_{\text {OUT }}\right\rangle=|3,0\rangle,|2,1\rangle,|1,2\rangle$, and $|0,3\rangle$ shown as solid green, red, blue, and magenta lines (line with the steepest slope, line with the second steepest slope, lower horizontal line, and upper horizontal line), respectively, for a fixed threshold $T=0.7$ as a function of the interaction $\lambda_{0}$. The crossing points of the energies $E_{\mathrm{TOT}}$ define critical interactions $\lambda_{c_{i}}$ where the energetically favorable final state of the dynamics is changing. (b) The nonescape probabilities $P_{\text {not }}^{x}(t, T=0.7)$ for different interactions $\lambda_{0}=0.5,1.0,30$ plotted from top to bottom as the solid red and blue and dotted blue lines, respectively. For $\lambda_{0}=0.5$ the final state is $|2,1\rangle$, whereas for $\lambda_{0}=1.0$ and 30 it is the state $|1,2\rangle$. The two horizontal dashed lines mark the respective nonescape probabilities $P_{\text {not }}^{x}=\frac{2}{3}$ and $P_{\text {not }}^{x}=\frac{1}{3}$. See the text for further discussion. All quantities shown are dimensionless.

closes the $|0,3\rangle$ channel, or, in other words, no simultaneous three-boson tunneling process occurs. The two-boson system remaining behind, $|2,0\rangle$, can eject the second boson, because it is higher in energy than the two-boson state $|1,1\rangle$. These two boson ejections will be seen in the momentum correlations discussed below. No further boson can be ejected, because for $T=0.7$ and any interaction the one-boson OUT state $|0,1\rangle$ is energetically higher than the IN state $|1,0\rangle$. All in all, the convergence of the nonescape probability to $\frac{1}{3}$ is understood.

As a final example we consider a much stronger interaction of $\lambda_{0}=30$. What should we expect? That the system has higher energy and therefore it tunnels faster. This is indeed the case [see Fig. 8(b)]. For such a strong interaction the initial three-boson state is fermionized, and one might expect the model Fig. 2 to become less accurate, because it is based on the neglect of the interactions outside. Still, the nonescape probability converges to $\frac{1}{3}$, indicating the counting statistics of the state $|1,2\rangle$, following the prediction of Fig. $8(\mathrm{~b})$. The findings suggest that the scheme for the control of the tunneling process in the presence of a threshold should hold for larger interaction strengths and for a larger number of bosons. Indeed, an application for a system of 101 interacting bosons will demonstrate this in the subsequent section.

We now briefly discuss other characteristics of the timedependent tunneling process when changing the interaction strength. As in Fig. 6, depletion and eventually fragmentation of the initially condensed system build up. If the interaction is stronger, this buildup naturally occurs faster. We have computed the numerically exact momentum distribution as a function of the interaction strength $\lambda_{0}$, and have explicitly verified that the model Eq. (8) performs well. Since the main information from the momentum distribution is encoded into the momentum correlation function $g^{(1)}$, we expand only on the latter.
Figure 9 shows $\left|g^{(1)}\left(k_{1}, k_{1}^{\prime}, t=800\right)\right|^{2}$ for a fixed threshold $T=0.7$ and interactions $\lambda_{0}=0.5,1.0$. We can see that on increasing the interaction $\lambda_{0}$ across the critical value for the availability of the counting statistics state $|1,2\rangle$, new lines appear. The ejected particles lose their coherence with the source at rest and among each other. Thus, the first-order momentum coherence (as well as the momentum distribution) can also be managed by the manipulation of $\lambda_{0}$, as they have been shown to be controlled by changing the threshold $T$. Of course, increasing the interaction has the "reverse" effect of increasing the threshold. The former favors counting statistics channels with more particles in the OUT region, whereas the latter favors those with more particles in the IN region; compare the sequences of panels in Figs. 7 and 9.

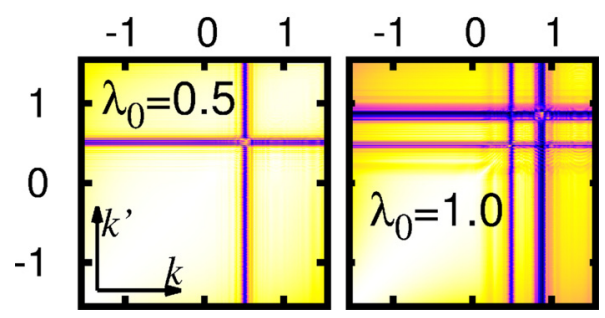

FIG. 9. (Color online) Coherence dynamics in the tunneling to open space of $N=3$ bosons as a function of the interaction strength $\lambda_{0}$. Shown is the single-particle momentum correlation function $\left|g^{(1)}\left(k^{\prime}, k ; t\right)\right|^{2}$ at time $t=800$ and a fixed threshold $T=0.7$ for $\lambda_{0}=$ $0.5,1.0$. White corresponds to full coherence, $\left|g^{(1)}\left(k^{\prime}, k ; t\right)\right|^{2}=1$, and black to full incoherence, $\left|g^{(1)}\left(k^{\prime}, k ; t\right)\right|^{2}=0$. The ejected particles lose their coherence with the source and with each other. The change of the final state is manifest in the emergence of a second line where coherence is lost $\left(\lambda_{0}=1.0\right)$. See the text for further discussion and complementary video in the Supplemental Material [60]. All quantities shown are dimensionless. 

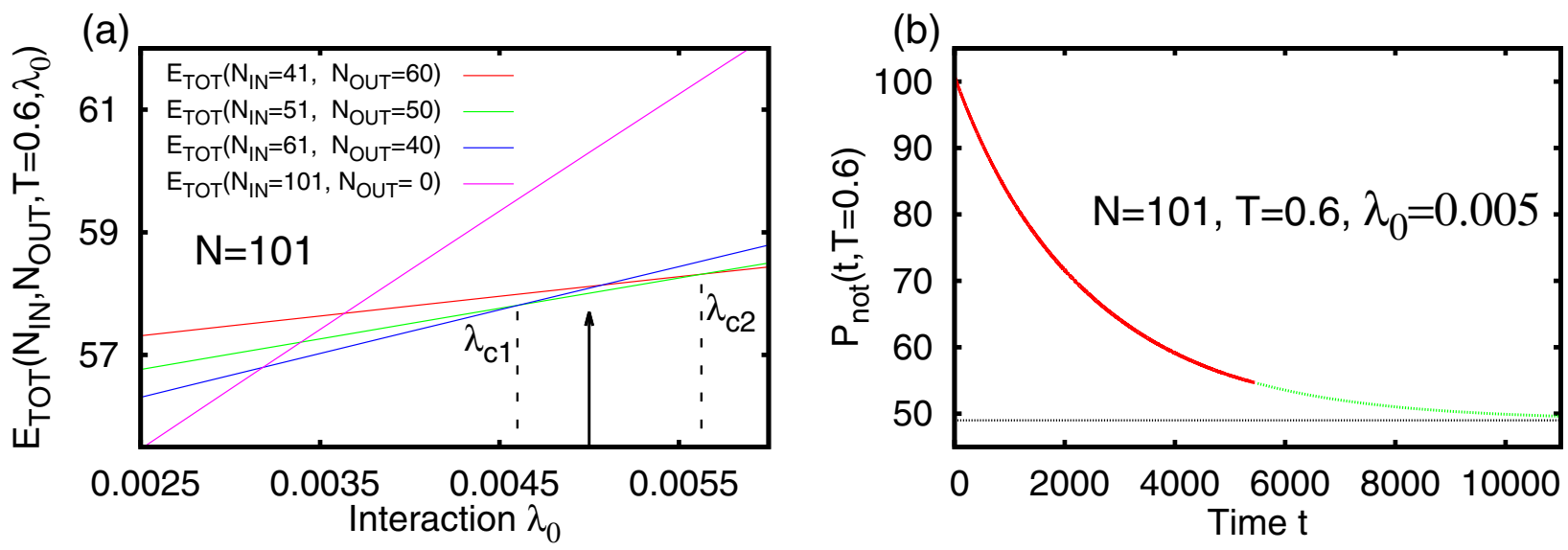

FIG. 10. (Color online) Controlling the tunneling to open space of an interacting 101-boson system. (a) Energies of selected final states $|41,60\rangle,|51,50\rangle$, and $|61,40\rangle$ for the threshold $T=0.6$ as a function of the interaction $\lambda_{0}$. For reference the energy of the initial state $|101,0\rangle$ is also depicted. When one tunes $\lambda_{0}$ such that it is in between the critical interactions $\lambda_{c 1}$ and $\lambda_{c 2}$ (marked by the black dashed vertical lines), the energy of the $|51,50\rangle$ state (green line) is lower than the energy of the $|41,60\rangle$ state (magenta line) and the $|61,40\rangle$ state (red line). An interaction chosen in this interval is expected to sustain the control objective of $N_{\mathrm{IN}} \approx 50$. The black arrow shows the interaction $\lambda_{0}=0.005$ chosen for the tunneling process. (b) The nonescape probability (red solid line). According to a least-squares fit (see green dotted line), the final state is $|49,52\rangle$ (corresponding to the horizontal dotted line) and hence in the range assessed from the energetics in panel (a). See the text for further discussion. All quantities shown are dimensionless.

\section{AN APPLICATION TO A SYSTEM OF 101 BOSONS}

Having learned the role of the threshold and interaction in controlling the tunneling to open space dynamics, we move to an application. We consider a system of initially trapped $N=101$ interacting bosons and aim at devising a scheme allowing roughly half of the bosons to tunnel out, or, equivalently, $N_{\text {IN }}=51$ bosons stay trapped. We first fix the threshold $T$ and then tune the interaction $\lambda_{0}$ appropriately. It is noteworthy that it is also possible to choose the reverse strategy (i.e., fixing the interaction and varying the threshold).

Figure 10(a) shows the energetics of the counting statistics states $|41,60\rangle,|51,50\rangle$, and $|61,40\rangle$ for the threshold $T=0.6$ as a function of $\lambda_{0}$, which are relevant for our analysis. We have identified a whole range of interaction strengths where of these states the counting statistics state $|51,50\rangle$ is lowest in energy [see Fig. 10(a)]. We expect an interaction chosen in this interval, say $\lambda_{0}=0.005$, to sustain the control objective of roughly half of the bosons tunneling out.

In Fig. 10(b) the survival probability of the system of $N=101$ interacting bosons tunneling to open space is computed numerically. We stress that this is a demanding time-dependent many-body task; see Appendix B. An analysis of the results indicates that the counting statistics converges to the state $|49,52\rangle$. This is in excellent agreement with our control objective.

The development of fragmentation and the characteristics of the momentum density and correlations resemble the ones analyzed above for a few bosons. Generally, when the number of particles is larger the distances between the momentum peaks decrease and the minima between the peaks become less pronounced. Hence, the distinct lines in the momentum density are not as clearly visible in the case of a large number of particles, similarly to the situation found in the absence of a threshold [35]. Still, the momenta at which the coherence is lost are well predicted by the model Eq. (8). To predict these momenta, one first determines the number of atoms $N_{\text {IN }}$ which is still bound for the chosen threshold and interaction. Subsequently, Eq. (8) is used to determine the momenta of the $N_{\text {OUT }}$ escaping particles.

\section{BRIEF SUMMARY AND OUTLOOK}

In the present work we have shown that one can fully control the number of ejected particles and their momentum density in the process of tunneling to open space. This can be achieved by manipulating the interplay of the threshold of the potential and the two-body interaction. We have exemplified and explicitly demonstrated this paradigm by investigating the tunneling to open space of interacting bosons in one spatial dimension. The investigation involved the recently developed MCTDHB method, and numerically exact results for two, three, and one hundred interacting bosons are reported. These results have been used as a benchmark for the numerical exactness of the recursive MCTDHB (R-MCTDHB) package, see Ref. [61].

The overall many-boson tunneling process has been shown to be made up of single-particle tunneling processes, which are well described using a transparent model (Fig. 2). An analysis of the energies of available decay channels together with the numerical results rule out the situation of two (or more) bosons tunneling together to open space.

Using the above-mentioned model, the many-boson tunneling process can be interpreted as follows. Let there be $N$ initially trapped bosons. The momentum of the first ejected boson draws from the chemical potential of the $N$-particle system. The available energy is used to overcome the threshold and the remainder transformed to kinetic energy of the boson outside the trap. The "fastest" peak in the momentum distribution corresponds to this kinetic energy. The momentum of the second ejected boson analogously draws from the 
chemical potential of the $(N-1)$-particle system. It is a bit slower. And so on, until a system of $N_{\mathrm{IN}}$ bosons remains bound and the rest $N_{\text {OUT }}$ have tunneled out.

At the momenta of the ejected bosons the system loses its coherence. Explicitly, the ejected bosons lose coherence between each other and with the "depleting" source of bosons in the trap. It means that one can control the structure of the coherence and correlations in the tunneling process by switching on or off certain processes with the interaction $\lambda_{0}$ and/or with the threshold $T$. Since the external potential and interparticle interactions can be controlled almost at will, the system's many-body tunneling dynamics is also under extensive control. Furthermore, the control schemes and found physics of the dynamics, especially in the exterior part of the potential, are similar to atom lasers (see, e.g., Refs. [3,62-64]) and could be useful to simulate and design their physical properties.

\section{ACKNOWLEDGMENTS}

Financial support by the DFG is gratefully acknowledged. A.U.J.L thanks the HGS MathComp and S.K. the Minerva
Foundation for financial support. Computation time on the Cray XE6 system Hermit and the NEC Nehalem cluster Laki at the Höchstleitsungsrechenzentrum Stuttgart and the bwGRiD-initiative in Esslingen, Ulm, Freiburg, and Stuttgart are gratefully acknowledged.

\section{APPENDIX A: DESIGN OF THE THRESHOLD POTENTIAL}

In the present work we investigate control mechanisms for the many-body tunneling process to open space. Since we wish to employ a one-particle potential $V(x)$ with a threshold, its design and numerical implementation are crucial. It should be (i) a smooth, continuous function which is (ii) identical to a parabolic trap in one region of space, and (iii) separated from the free, asymptotic part by a barrier. Furthermore, (iv) the value $T$ of the threshold potential in this free, asymptotic region of space should be easy to modify.

In order to be flexible with the value $T$ of the threshold it is practical to use a smooth polynomial continuation of the harmonic trap $V_{h}(x)=\frac{1}{2} x^{2}$ from $x_{c 1}=2$ to $x_{c 2}=4$ (see Fig. 1). The details of the polynomial $P(x)$ are given below. We first present the potential obtained in its final form:

$$
V(x, t)= \begin{cases}\frac{1}{2} x^{2}, & t=0 \\ \Theta\left(x_{c 1}-x\right) \frac{1}{2} x^{2}+\Theta\left(x-x_{c 1}\right) \Theta\left(x_{c 2}-x\right) P(x)+\Theta\left(x-x_{c 2}\right) T, & t>0\end{cases}
$$

Here, $\Theta(\cdot)$ is the Heaviside step function. Plots of the potential in Eq. (A1) with various values of $T$ are depicted in Fig. 1. The time variable in Eq. (A1) implies that at $t=0^{+}$the harmonic potential is suddenly changed to the open trap. We have checked that, because of the smoothness of the potential [see ingredients (i) and (ii) above], the sudden change in the potential does hardly change the energy or shape of the initially trapped wave function.

By using a polynomial continuation to the threshold, the position of the maximum of the potential, $x_{m}$, depends on the threshold $T$ as follows:

$$
x_{m}(T)=2+\frac{1}{3-\frac{3}{4} T}
$$

see Fig. 1.

The polynomial $P(x)$ is determined as follows. There are four constraints to the polynomial continuation, namely, that both the polynomial itself and its first derivative have to be equal to the value and first derivative of the neighboring potential, at both $x_{c 1}=2$ and $x_{c 2}=4$ (see Fig. 1). Therefore, a polynomial of at least third order with four coefficients $A, B, C, D$ is required:

$$
P(x)=A x^{3}+B x^{2}+C x+D .
$$

With the constraints

$$
\begin{gathered}
P\left(x_{c 1}\right)=A x_{c 1}^{3}+B x_{c 1}^{2}+C x_{c 1}+D=V_{h}\left(x_{c 1}\right)=2, \quad(\mathrm{~A} 4) \\
\left.\frac{d}{d x} P(x)\right|_{x=x_{c 1}}=3 A x_{c 1}^{2}+2 B x_{c 1}+C=\left.\frac{d}{d x} V_{h}(x)\right|_{x=x_{c 1}}=2
\end{gathered}
$$

for the connection at $x_{c 1}$ to the harmonic trapping potential $V_{h}(x)$ and

$$
\begin{gathered}
P\left(x_{c 2}\right)=A x_{c 2}^{3}+B x_{c 2}^{2}+C x_{c 2}+D=T, \\
\left.\frac{d}{d x} P(x)\right|_{x=x_{c 2}}=3 A x_{c 2}^{2}+2 B x_{c 2}+C=\frac{d}{d x} T=0
\end{gathered}
$$

for the connection to the constant threshold $T$ at $x_{c 2}$. From these four equations the coefficients $A(T), B(T), C(T), D(T)$ can easily be obtained; see Table I. One can hence control the threshold $T$ arbitrarily while maintaining a smooth potential.

\section{APPENDIX B: THE MULTICONFIGURATIONAL TIME-DEPENDENT HARTREE METHOD FOR BOSONS AND COMPUTATIONAL DETAILS}

The MCTDHB method $[47,48]$ is well documented in the literature [56,57], and is capable of providing numerically exact solutions of the time-dependent many-boson Schrödinger equation (TDSE) (see Ref. [54]). We briefly capture its

TABLE I. Parameters of the potential with a threshold $T$ entering Eqs. (A3)-(A7).

\begin{tabular}{lc}
\hline \hline Coefficient & Value at $T=0.5$ \\
\hline$A(T)=-\frac{1}{4} T+1$ & 0.875 \\
$B(T)=\frac{9}{4} T-\frac{19}{2}$ & 8.375 \\
$C(T)=-6 T+28$ & 25.0 \\
$D(T)=5 T-24$ & 21.5 \\
\hline \hline
\end{tabular}


essence and usage in the present work. The MCTDHB method relies on expanding the wave function with multiple, timedependent configurations $|\vec{n} ; t\rangle$ weighted with time-dependent coefficients $C_{\vec{n}}(t)$ :

$$
|\Psi\rangle=\sum_{\vec{n}} C_{\vec{n}}(t)|\vec{n} ; t\rangle .
$$

The configurations constitute a many-body basis built by applying creation operators in at most $M$ single-particle time-adaptive states to the quantum mechanical vacuum $|\mathrm{vac}\rangle$ :

$$
\begin{aligned}
|\vec{n} ; t\rangle & =\left|n_{1}, n_{2}, \ldots, n_{M} ; t\right\rangle \\
& =\frac{1}{\sqrt{\prod_{i=1}^{M} n_{i} !}}\left[\hat{b}_{1}^{\dagger}(t)\right]^{n_{1}}\left[\hat{b}_{2}^{\dagger}(t)\right]^{n_{2}} \cdots\left[\hat{b}_{M}^{\dagger}(t)\right]^{n_{M}}|\mathrm{vac}\rangle
\end{aligned}
$$

The MCTDHB equations of motion are obtained by tackling the TDSE with the time-dependent variational principle and requiring the stationarity of the resulting functional action when varying the coefficients $C_{\vec{n}}(t)$ and the single-particle states $\hat{b}_{k}^{\dagger}(t)$ (see Ref. [48]). The $\left(\begin{array}{c}N+M-1 \\ N\end{array}\right)$ linear equations of motion for the coefficients are coupled to the $M$ nonlinear integro-differential equations of motion of the orbitals. Since the derivation is variational and the basis used is a formally complete set in the limit $M \rightarrow \infty$, convergence with respect to the number of orbitals implies convergence to the exact solution of the TDSE for the problem under consideration [54]. The use of time-adaptive orbitals is of key importance for the achievement of numerical exactness: A much smaller number of time-adaptive orbitals is needed to achieve the same level of accuracy as compared to the number of basis functions in a static, time-independent basis [54].

In the present work the MCTDHB software package [49] was employed to obtain such converged solutions of the TDSE. The computations used grids of sizes of up to $[-5 ; 7465]$ in dimensionless units, represented by up to $2^{16}=65536$ timeindependent basis functions (grid points) with up to $M=14$ time-adaptive orbitals.
[1] O. Morsch and M. K. Oberthaler, Rev. Mod. Phys. 78, 179 (2006).

[2] M. Greiner, O. Mandel, T. Esslinger, T. W. Hänsch, and I. Bloch, Nature (London) 415, 39 (2002).

[3] W. Ketterle, Rev. Mod. Phys. 74, 1131 (2002).

[4] M. H. Anderson, J. R. Ensher, M. R. Matthews, C. E. Wiemann, and E. A. Cornell, Science 269, 198 (1995).

[5] C. C. Bradley, C. A. Sackett, J. J. Tollett, and R. G. Hulet, Phys. Rev. Lett. 75, 1687 (1995).

[6] K. Henderson, C. Ryu, C. MacCormick, and M. G. Boshier, New J. Phys. 11, 043030 (2009).

[7] C. Chin, R. Grimm, P. Julienne, and E. Tiesinga, Rev. Mod. Phys. 82, 1225 (2010).

[8] A. Görlitz, J. M. Vogels, A. E. Leanhardt, C. Raman, T. L. Gustavson, J. R. Abo-Shaeer, A. P. Chikkatur, S. Gupta, S. Inouye, T. Rosenband, and W. Ketterle, Phys. Rev. Lett. 87, 130402 (2001).

[9] F. Schreck, L. Khaykovich, K. L. Corwin, G. Ferrari, T. Bourdel, J. Cubizolles, and C. Salomon, Phys. Rev. Lett. 87, 080403 (2001).

[10] M. Greiner, I. Bloch, O. Mandel, T. W. Hänsch, and T. Esslinger, Phys. Rev. Lett. 87, 160405 (2001).

[11] I. Bloch, J. Dalibard, and W. Zwerger, Rev. Mod. Phys. 80, 885 (2008).

[12] A. Hemmerich and C. M. Smith, Phys. Rev. Lett. 99, 113002 (2007).

[13] H. Pu, W. Zhang, and P. Meystre, Phys. Rev. Lett. 87, 140405 (2001).

[14] O. Lahav, A. Itah, A. Blumkin, C. Gordon, S. Rinott, A. Zayats, and J. Steinhauer, Phys. Rev. Lett. 105, 240401 (2010).

[15] J. Macher and R. Parentani, Phys. Rev. A 80, 043601 (2009).

[16] J.-C. Jaskula, G. B. Partridge, M. Bonneau, R. Lopes, J. Ruaudel, D. Boiron, and C. I. Westbrook, Phys. Rev. Lett. 109, 220401 (2012).

[17] G. Gamow, Z. Phys. 51, 204 (1928).

[18] B. S. Bhandari, Phys. Rev. Lett. 66, 1034 (1991).
[19] T. N. Balantekin, Rev. Mod. Phys. 70, 77 (1998).

[20] W. J. Keller and J. Weiner, Phys. Rev. A 29, 2943 (1984).

[21] M. Vatasescu, O. Dulieu, C. Amiot, D. Comparat, C. Drag, V. Kokoouline, F. Masnou-Seeuws, and P. Pillet, Phys. Rev. A 61, 044701 (2000).

[22] M. Razavy, Quantum Theory of Tunneling (World Scientific, Singapore, 2003).

[23] H. A. Kramers, Z. Phys. A 39, 828 (1926).

[24] R. W. Gurney and E. U. Condon, Nature (London) 122, 439 (1928).

[25] R. W. Gurney and E. U. Condon, Phys. Rev. 33, 127 (1929).

[26] S. Kim and J. Brand, J. Phys. B 44, 195301 (2011).

[27] J. A. Glick and L. D. Carr, arXiv:1105.5164 [cond-mat.quantgas].

[28] A. del Campo, F. Delgado, G. García-Calderón, J. G. Muga, and M. G. Raizen, Phys. Rev. A 74, 013605 (2006).

[29] A. del Campo and J. Muga, Europhys. Lett. 6, 965 (2006).

[30] S. Hunn, K. Zimmermann, M. Hiller, and A. Buchleitner, Phys. Rev. A 87, 043626 (2013).

[31] L. D. Carr, M. J. Holland, and B. A. Malomed, J. Phys. B 38, 3217 (2005).

[32] N. Moiseyev and L. S. Cederbaum, Phys. Rev. A 72, 033605 (2005).

[33] P. Schlagheck and S. Wimberger, Appl. Phys. B: Lasers Opt. 86, 385 (2007).

[34] A. U. J. Lode, A. I. Streltsov, O. E. Alon, H.-D. Meyer, and L. S. Cederbaum, J. Phys. B 42, 044018 (2009); 43, 029802 (2010).

[35] A. U. J. Lode, A. I. Streltsov, K. Sakmann, O. E. Alon, and L. S. Cederbaum, Proc. Natl. Acad. Sci. USA 109, 13521 (2012).

[36] P. Nozières and D. Saint James, J. Phys. (France) 43, 1133 (1982).

[37] P. Nozières, in Bose-Einstein Condensation, edited by A. Griffin, D. W. Snoke, and S. Stringari (Cambridge University Press, Cambridge, England, 1996).

[38] R. W. Spekkens and J. E. Sipe, Phys. Rev. A 59, 3868 (1999). 
[39] O. E. Alon and L. S. Cederbaum, Phys. Rev. Lett. 95, 140402 (2005).

[40] S. Klaiman, N. Moiseyev, and L. S. Cederbaum, Phys. Rev. A 73, 013622 (2006).

[41] E. J. Müller, T.-L. Ho, M. Ueda, and G. Baym, Phys. Rev. A 74, 033612 (2006).

[42] A. I. Streltsov, O. E. Alon, and L. S. Cederbaum, Phys. Rev. A 73, 063626 (2006).

[43] U. Titulaer and R. Glauber, Phys. Rev. 140, B676 (1965).

[44] R. J. Glauber, Phys. Rev. 130, 2529 (1963).

[45] M. Naraschewski and R. J. Glauber, Phys. Rev. A 59, 4595 (1999).

[46] K. Sakmann, A. I. Streltsov, O. E. Alon, and L. S. Cederbaum, Phys. Rev. A 78, 023615 (2008).

[47] A. I. Streltsov, O. E. Alon, and L. S. Cederbaum, Phys. Rev. Lett. 99, 030402 (2007).

[48] O. E. Alon, A. I. Streltsov, and L. S. Cederbaum, Phys. Rev. A 77, 033613 (2008).

[49] A. I. Streltsov, K. Sakmann, A. U. J. Lode, O. E. Alon, and L. S. Cederbaum, The Multiconfigurational Time-Dependent Hartree for Bosons package, version 2.3, Heidelberg, 2013, http://MCTDHB.org.

[50] K. Sakmann, A. I. Streltsov, O. E. Alon, and L. S. Cederbaum, Phys. Rev. Lett. 103, 220601 (2009).

[51] K. Sakmann, A. I. Streltsov, O. E. Alon, and L. S. Cederbaum, Phys. Rev. A 82, 013620 (2010).

[52] J. Grond, T. Betz, U. Hohenester, N. J. Mauser, J. Schmiedmayer, and T. Schumm, New J. Phys. 13, 065026 (2011).
[53] I. Březinová, A. U. J. Lode, A. I. Streltsov, O. E. Alon, L. S. Cederbaum, and J. Burgdörfer, Phys. Rev. A 86, 013630 (2012).

[54] A. U. J. Lode, K. Sakmann, O. E. Alon, L. S. Cederbaum, and A. I. Streltsov, Phys. Rev. A 86, 063606 (2012).

[55] M. Heimsoth, D. Hochstuhl, C. E. Creffield, L. D. Carr, and F. Sols, New J. Phys. 15, 103006 (2013).

[56] Multidimensional Quantum Dynamics: MCTDH Theory and Applications, edited by H.-D. Meyer, F. Gatti, and G. A. Worth (Wiley-VCH, Weinheim, 2009).

[57] Quantum Gases: Finite Temperature and Non-Equilibrium Dynamics, edited by N. P. Proukakis, S. A. Gardiner, M. J. Davis, and M. H. Szymanska, Cold Atoms Series Vol. 1 (Imperial College Press, London, 2013).

[58] A. Coleman and V. Yukalov, Reduced Density Matrices: Coulson's Challenge (Springer, Heidelberg, 2000).

[59] O. Penrose and L. Onsager, Phys. Rev. 104, 576 (1956).

[60] See Supplemental Material at http://link.aps.org/supplemental/ 10.1103/PhysRevA.89.053620 for videos of the momentum density and correlations in the tunneling to open space dynamics.

[61] A. U. J. Lode and M. C. Tsatsos, The Recursive Multiconfigurational Time-Dependent Hartree for Bosons Package (2014), http://ultracold.org; http://r-mctdhb.org; http://schroedinger.org.

[62] I. Bloch, T. W. Hänsch, and T. Esslinger, Phys. Rev. Lett. 82, 3008 (1999).

[63] A. Öttl, S. Ritter, M. Köhl, and T. Esslinger, Phys. Rev. Lett. 95, 090404 (2005).

[64] M. Köhl, T. Busch, K. Mølmer, T. W. Hänsch, and T. Esslinger, Phys. Rev. A 72, 063618 (2005). 